\title{
ANÁLISE MODAL DE COMPONENTES DO SISTEMA DE EXAUSTÃO: UM ESTUDO DE CASO CONSIDERANDO AS ALTERNATIVAS DAS FREQUÊNCIAS NATURAIS
}

\author{
Evandro Secchi de Oliveira ${ }^{1}$ e Roderley Camargo ${ }^{2}$ \\ ${ }^{1}$ UNISAL Centro Universitário Salesiano de São Paulo \\ ${ }^{1}$ Bosal do Brasil Ltda \\ ${ }^{2}$ Robert Bosch Ltda
}

E-mails: evandro.oliveira@bosal.com.br, roderley.camargo@br.bosch.com

\section{RESUMO}

Quando o veículo está em movimento, o sistema de exaustão fica sob influência de todas as forças transmitidas para ele. Estas solicitações dinâmicas podem ser de vibração, ou de um grande deslocamento, causado por irregularidades da estrada, quando o veículo por exemplo, passa em um grande desnível. Neste caso, o impacto ocasionado pelo contato do pneu com a estrada, transfere uma parte desta força ao escapamento, fazendo com que haja um grande deslocamento, e consequentemente, gerando tensões em seus componentes. Sob este ponto de vista, realizou-se neste trabalho, estudos de simulações via método dos elementos finitos, identificando as regiões de concentração de tensões, no instante em que o veículo sofre um impacto, ocasionado por desníveis na pista. Com isto, foi analisado o projeto e verificado se o mesmo, está adequado aos critérios de falha adotados pela engenharia do produto. Foram inseridas acelerações em cada eixo do escapamento, para simular a força transmitida por um impacto, forças estas da ordem de $5 \mathrm{G}$ nas direções "X" e "Y", e 10G na direção "Z" do plano cartesiano. Estas informações foram adquiridas pela montadora, por meio de acelerômetros localizados no sistema de exaustão. Como critério de falha, definiu-se o limite de $700 \mathrm{Mpa}$ de tensão. A análise da simulação computacional foi útil, pois, identificou-se a região do produto com tensões fora da especificação técnica, sendo possível o estudo e a avaliação funcional deste requisito. $\mathrm{O}$ desdobramento e detalhamento desta analise está descrito ao longo deste trabalho, indicando também as ações preventivas sob o ponto de vista das frequências naturais.

\section{INTRODUÇÂO}

O conjunto formado pelo sistema de exaustão automotivo, utilizado nos veículos de ciclo OTTO, está localizado na parte de baixo do veículo e interligado ao motor. Umas das finalidades deste conjunto, é a atenuação dos ruídos provenientes do motor, assim como, prover a diminuição dos gases nocivos à saúde, por intermédio de um catalisador. Também faz parte da função do sistema de exaustão, o arrefecimento dos gases de saída, antes da liberação para a atmosfera, como forma de contribuição aos compromissos ecológicos sustentáveis.

Este trabalho tem por finalidade, a determinação das acelerações que o sistema sofre, durante um impacto, no momento em que o veículo automotivo passar em um desnível da estrada. 
Para isto, realizou-se estudos empíricos nos veículos por parte da montadora, em que foram utilizados acelerômetros, instalados no sistema de escapamento.

Neste estudo, utilizou-se um sistema de exaustão de um carro compacto, com motorização de 4 cilindros, 1.0 litro de cilindrada. Adotou-se como critério de falha, que as tensões necessitam ser inferiores a 700Mpa, sendo considerado ideal, tensões abaixo de 500Mpa. Tensões localizadas entre as faixas de 500MPa a 700Mpa, devem ser avaliadas pela engenharia do produto, a ocorrência de risco para o projeto como um todo.

Nos dados de entrada do software, foram levados em considerações as especificações do material do hanger, dos tubos onde os mesmos são soldados e as especificações das próprias soldas.

Com o software Hypermesh, foram criadas as malhas e as condições de contorno, e inserido todas as informações dos materiais, quais sejam: Espessura, Modulo de Young, Densidade e Coeficiente de Poisson. Para rodar a simulação e obter os resultados de tensões, empregou-se os algoritmos de convergência do solver ABAQUS.

No final, utilizou-se o software Hyperview para a análise dos dados de saída do Abaqus e a geração dos relatórios correlatos.

Estes relatórios são de extrema importância para a estratégia do engenheiro do produto, pois com isso, o mesmo poderá verificar o projeto e fazer modificações, ainda na fase de pré desenvolvimento do produto. Este tipo de ação preventiva de engenharia, colabora para uma maior assepsia de projeto, dando sustentabilidade aos argumentos ora teorizados, e na direção do projeto enxuto.

Desta forma, a construção dos protótipos e demais testes físicos ficam simplificados, e tem-se uma garantia maior do sucesso da prova prática e testes funcionais.

Um outro beneficio da simulação computacional, esta relacionado a redução de custo e possível aumento da resistência estrutural do componente.

O intuito deste trabalho é iniciar um estudo pela técnica dos elementos finitos, que tem por finalidade identificar as regiões de concentração de tensão, e analisar se o material está adequado ao projeto, assim como se haverá a necessidade da criação de algum tipo de reforço estrutural nas regiões denotadas.

\section{INFORMAÇÔES GERAIS}

Para o desenvolvimento de produtos inovadores e bem-sucedidos, no atual cenário global e competitivo, há que se ter recursos de informática cientifica e fazer uso intensivo de simulação. Neste senso, para que a empresa sobreviva neste ambiente de competição, a simulação deve fazer parte do projeto, já na fase inicial do processo de desenvolvimento do produto [1]. A capacidade de analisar as características multicorpos do desempenho físico de um projeto, antes de criar um primeiro protótipo, pode aumentar de forma significativa a produtividade do mesmo.

As empresas precisam de ferramentas de simulação robustas, o suficiente para superar com eficácia, as demandas de prazo, custo e qualidade final do produto [2]. A tecnologia de simulação permite a utilização da modelagem matemática, baseada em computador, para 
aproximar e simular os fenômenos complexos do universo físico, tendo como dados de entrada, o modelo CAD 3D [3]. Com o modelo matemático CAD, enviado pela montadora, inicia-se o processo de construção da malha de elementos finitos, em que é possível indicar para cada parte da geometria $\mathrm{CAD}$, o tipo de elemento e tamanho adequado para cada região geométrica. Este processo é o inicio da formulação CAE, via métodos dos elementos finitos. Depois de inseridos todos os dados, é analisada a informação de saída, gerada pelo solver, validando ou reprovando o design atual. As conclusões e desdobramentos deste trabalho estão destacados no tópico estudo de caso.

\subsection{METODOLOGIA}

Empregou-se a metodologia FEA (Finite Element Analyze) aplicada à análise de frequências naturais, dos principais componentes: Ganchos de fixação, Atenuador de ruído e Silenciador.

Nesta técnica, a partir da matriz de deslocamentos dos nós e da matriz de rigidez, foi possível verificar as regiões de tensões no material, e a tendência de deformação. Foi utilizado um pacote de softwares orientados ao pré-processamento, cálculo e geração de relatórios. Este software de CAE, utiliza modelos matemáticos digitais 3D, para simular fenômenos físicos reais, via métodos numéricos convergentes [4].

Para a análise estática, considerou-se as tensões de Von Mises e o deslocamento, no intervalo de validação da lei de Hooke, dentro da fase elástica [5].

Assim, três etapas complementam este método: Pré-Processamento, Cálculo e Relatórios [6].

Na fase de Pré-Processamento, foi utilizado o software Hypermesh onde são considerados os seguintes requisitos: Geometria, Material, Forças aplicadas, Restrições, Propriedades físicas e Elementos.

Na fase de Cálculo, foi empregado o software ABAQUS que utiliza algoritmos internos, para a resolução matemática do problema proposto.

Na fase de relatórios, foi utilizado o software Hyperview considerando os resultados: Tensão, Deslocamento, Deformação e Tomografia virtual, evidenciando as isosurfaces. Neste momento, a intenção é convalidar estes resultados conforme os critérios adotados e citados anteriormente.

\subsection{FUNDAMENTAÇÂO TEÓRICA}

Com o intuito de comprovar a eficácia da simulação computacional, e visando também prever uma possível falha de função, a teoria matemática consiste em gerar uma matriz de identificação. Esta matriz objetiva identificar todos os nós gerados pela malha, em uma coordenada, e correlacionar com as forças aplicadas em cada nó, juntamente com a matriz de rigidez do conjunto [7].

A teoria matemática a ser comprovada é: $\mathrm{E}=\sigma / \varepsilon$, no limite da fase elástica, considerando o coeficiente de segurança. A resposta da estrutura as solicitações virtuais, serão expressas em tensões de Von Mises (MPa) e displacement $(\mathrm{mm})$, seguida de otimização topológica do conjunto formado pelo produto. 
A equação básica da análise estática é [8]:

$$
[\mathrm{K}\}\{\mathrm{u}\}=\{\mathrm{F}\}
$$

Em que:

"K" é a matriz de rigidez do sistema gerada automaticamente pelo ABAQUS, com base na geometria e propriedades aplicadas.

"F" é o vetor de forças aplicadas (o qual é especificado).

"u" é o vetor de deslocamentos que são computados pelo ABAQUS (elementos de força, tensão, reação, etc.).

Para a análise modal, tem-se [9]:

$$
[\mathrm{K}-\mathrm{M} . \lambda \mathrm{i}]\{\Phi \mathrm{i}\}=0
$$

Em que:

$K$ é a matriz de rigidez do sistema (propriedades de inércia).

$M$ é a matriz de massa do sistema (gerada automaticamente pelo ABAQUS com base na geometria e propriedades elásticas).

$\lambda i$ e $\Phi i$ são os auto valores a serem computados, onde $\lambda i$ é o auto valor e $\Phi i$ é o vetor de auto valor ou modal shape.

\section{ARQUITETURA BÁSICA DO MÉTODO DOS ELEMENTOS FINITOS}

A confiabilidade dos resultados obtidos, a partir do uso da metodologia FEA, se comparada aos diversos métodos empregados, se resume na facilidade de interface com o software de analise. O programa de elementos finitos busca na simplificação de operação, uma maneira de adequar os recursos de equações matemáticas e orientá-las ao objeto de analise [7].

Assim, três etapas contemplam uma programação via FEA, como forma de orientação a lógica computacional, quais sejam [10]:

\section{$P R E P R O C E S S I N G \rightarrow$ SOLVER $\rightarrow$ REPORT}

A fase de Preprocessing contempla os dados do material e os requisitos do projeto, quais sejam: Geometria, Material, Forças, Restrições e Tipos de elementos. Nesta fase, é de suma importância a representação gráfica da geometria do modelo CAD, com os principais detalhes construtivos, envolvendo a fabricação, montagem e o projeto de fato. Também é importante a entrada dos dados do material a ser utilizado na análise. Faz parte deste tópico: Módulo de Young, Coeficiente de Poisson e Densidade. Além disso, a leitura das condições de contorno 
pode ser feita nesta fase, assim como as condições de carregamento, também são introduzidas nesta etapa.

A Figura 1 ilustra a arquitetura computacional básica da análise FEA, contemplando as três etapas: Preprocessing, Solver e Report.

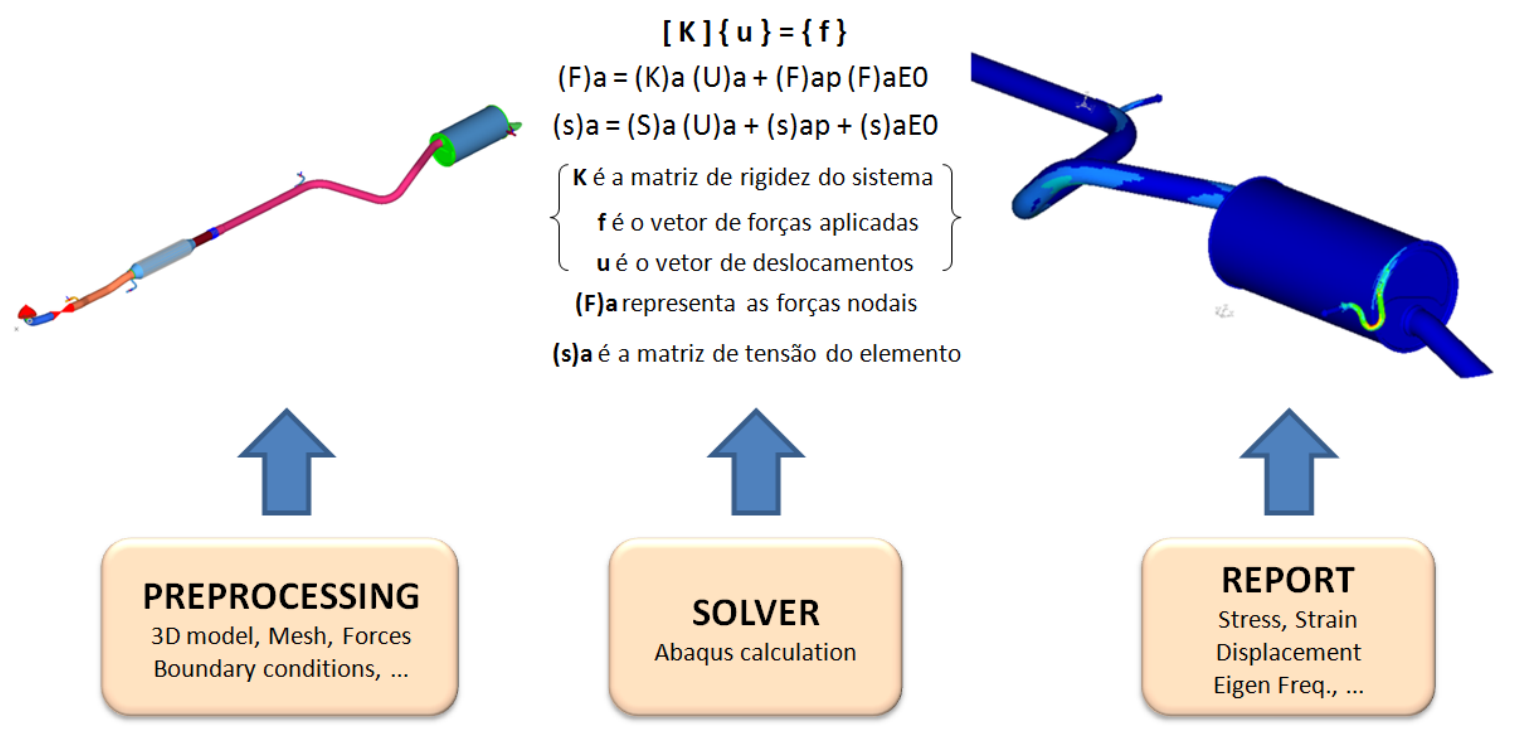

Figura 1 - Arquitetura computacional do método FEA

A fase do Preprocessing diz respeito a preparação da malha e dos elementos necessários, para discretizar o modelo matemático e iniciar os cálculos. Nesta fase, também é organizado toda as propriedades dos componentes, indicando se este foi construído com chapa, sólido maciço ou sólido de geometria variada. Também é indicado os parâmetros em que o solver irá trabalhar, como por exemplo, se a analise é estática, dinâmica ou se irá obedecer uma curva de carga, etc.

Na fase de Solver, é utilizado o algoritmo interno do ABAQUS, para a solução do sistema, e tem-se o resultado da análise estrutural, em diversos formatos de saída, de acordo com o critério de falha determinado no escopo da analise. São exemplos de saída de resultados: Tensões, Deformações, Deslocamentos, Gráficos, Diagramas, Temperaturas, Pressões entre outros.

Na fase de saída gráfica dos Resultados, - incluindo os aspectos de simulação - dão ao engenheiro analista, a noção exata do que está ocorrendo em termos de realidade aumentada, e convergindo para uma solução computacional robusta, no que se refere as condições de entrada [10].

\subsection{DEFINIÇÂO DA TÉCNICA DOS ELEMENTOS FINITOS}

A análise dos elementos finitos é uma ferramenta poderosa para se determinar as tensões e as deflexões, numa estrutura complexa demais para a análise clássica.

É uma técnica matemática que consiste em discretizar uma estrutura, - modelo matemático tridimensional - em elementos paramétricos ou isoparamétricos conhecidos, e conectados por 
"nós". As propriedades dos materiais e elementos são especificados afim de representarem as propriedades físicas do modelo, conforme ilustrado na Figura 2 [11].

Essencialmente, a técnica divide geometricamente uma estrutura, em pequenos elementos com características facilmente definidas de tensão e deflexão.

A Figura 2 ilustra uma estrutura e suas partes, composta por elementos e nós.

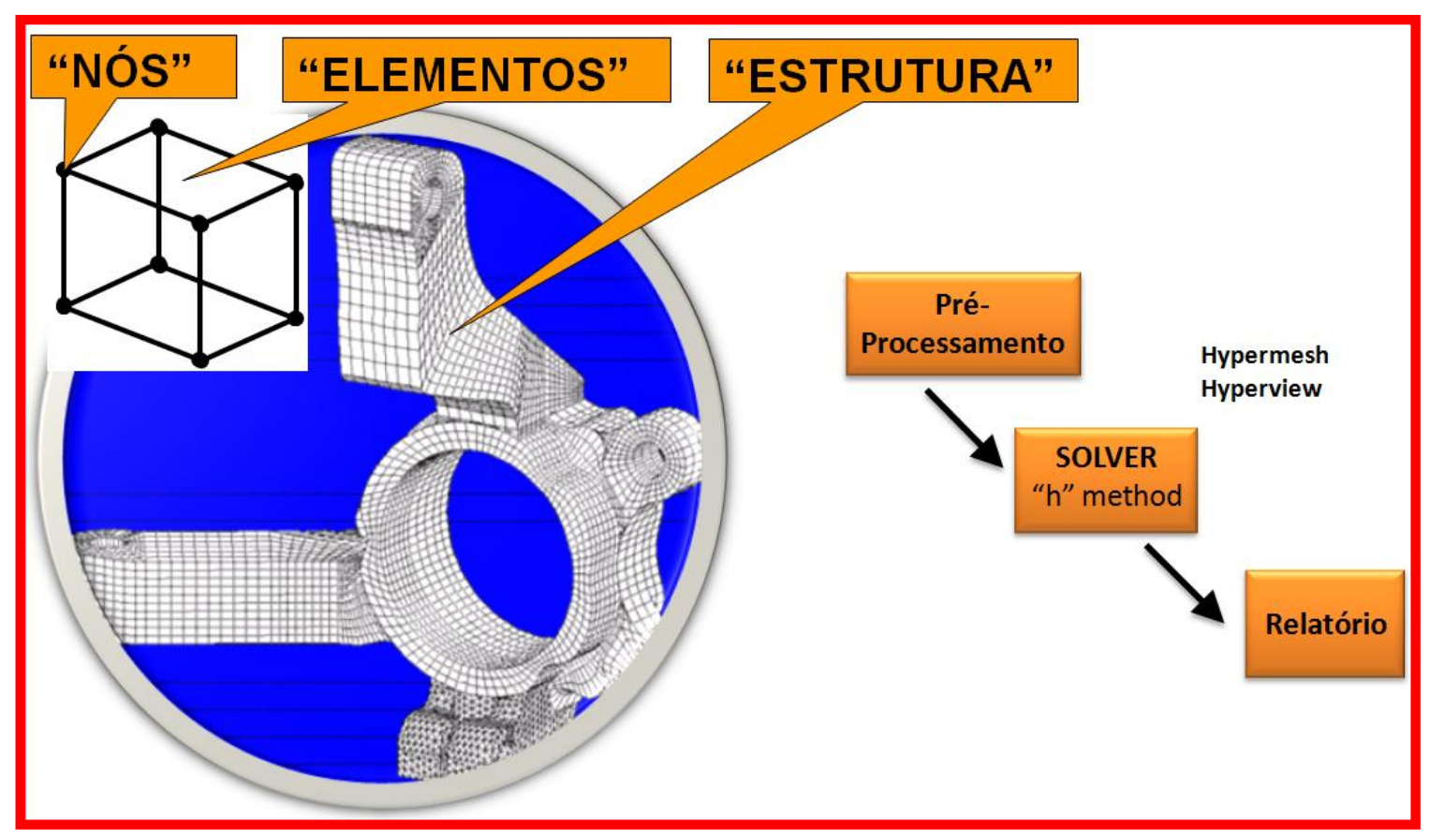

Figura 2 - Definição de estrutura, elementos e "nós"

Também há que se envolver todo o modelo matemático, inserindo as mesmas condições de contorno, consideradas no protótipo, e refinando a malha nos locais em que são mais relevantes, para que o resultado desta análise venha a convergir com o resultado do teste. Ao inserir a localização e a intensidade da carga, os nós gerados inicialmente sofrerão uma deformação, ou seja, um deslocamento entre os próprios nós.

O solver irá interpretar estes deslocamentos, tratando cada nó como um vetor, e a partir daí, cria-se uma matriz que por sua vez, indica qual a tensão gerada em cada nó que foi deslocada, em função da carga aplicada.

\subsection{ENTENDENDO A TEORIA "FEA"}

As condições de contorno são especificadas de acordo como o tipo de vinculação do problema, obedecendo as regras da mecânica dos sólidos [5], e da resultante do vetor das forças atuantes. A análise estrutural FEA, deve satisfazer os seguintes requisitos [12] e [13]:

- $\quad$ Obter equilíbrio entre: Forças e Momentos;

- Relacionar de forma compatível: Deformação x Deslocamento;

- Relacionar em forma de regra constitutiva: Tensão x Deformação. 
As condições acima, são aplicadas para gerar um sistema de equações, em que os deslocamentos são desconhecidos.

\subsection{MÉTRICAS DA ANÁLISE FEA}

Para simular com precisão, acuracidade e eficácia a complexidade dos fenômenos físicos do mundo real, e por consequência tratar seus efeitos nos projetos, há que se considerar:

- Compatibilidade: Correlação dos deslocamentos dos "nós" externos, fazendo uma solução convergente com a deformação dos elementos;

- Relação "Força/Deslocamento" se estabelece entre: "Elemento end force" e "Elemento end deformation" e também entre as "Forças Nodais e Deslocamentos";

- Equilíbrio imposto entre: Forças Internas e Externas dos Nós.

\subsection{EQUAÇÂO CONSTITUTIVA REDUZIDA}

A tensão em um ponto, tem que ser representada por um tensor, para poder expressar o vetor força em qualquer direção. Para o estudo do estado de tensões, no interior do corpo deformado, isola-se um elemento infinitesimal de um volume de dimensões: $\mathrm{x}, \mathrm{y}, \mathrm{z}$, conforme ilustrado na Figura 3 [14].

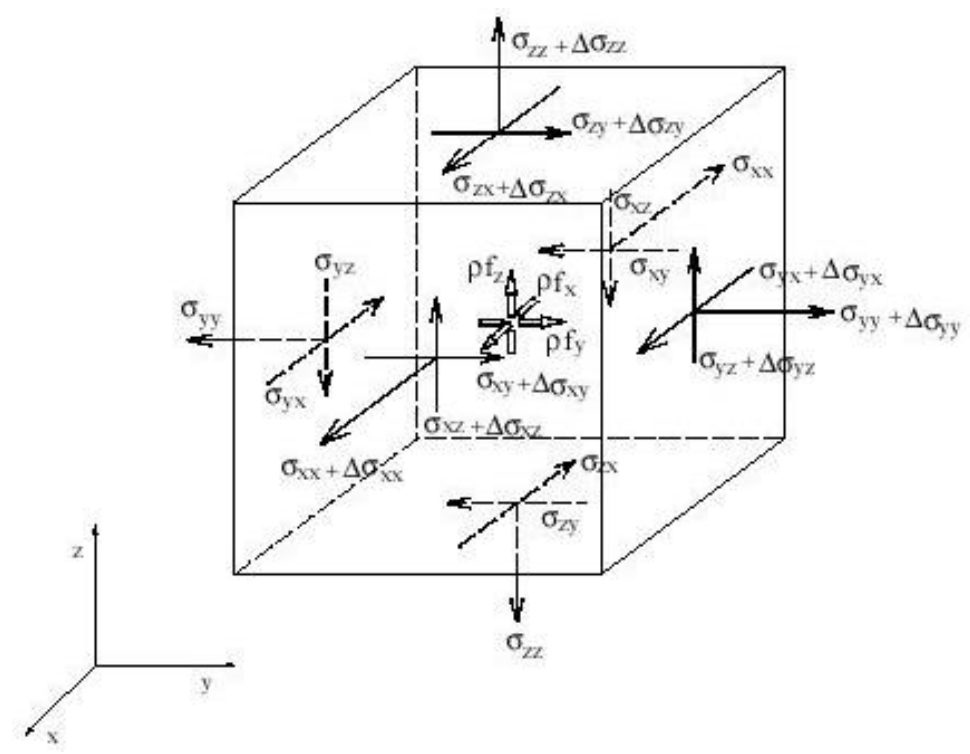

Figura 3 - Estado de tensões em um elemento infinitesimal [14]

A matriz de rigidez [K] é usada para relacionar FORÇA NODAL com DESLOCAMENTO NODAL.

Sendo os $\mathrm{K}_{\mathrm{ij}}$ os coeficientes de influência de rigidez (relaciona a força da constraint no DOF_i (Degree of Freedom), o termo típico $K_{i j}$ é definido como uma força no i-ésimo nó devido a um deslocamento unitário no j-ésimo nó, sendo os demais deslocamentos nulos.

Ainda conforme [14], as equações constitutivas (ou equações tensões-deformações) para sólidos elásticos lineares homogêneos, são dadas pela Lei de Hooke generalizada para materiais isotrópicos, quais sejam: 


$$
\begin{aligned}
& \sigma \mathrm{xx}=(\lambda+2 \mathrm{G}) \varepsilon \mathrm{xx}+\lambda \varepsilon \mathrm{yy}+\lambda \varepsilon \mathrm{zz} \\
& \sigma \mathrm{yy}=\lambda \varepsilon \mathrm{xx}+(\lambda+2 \mathrm{G}) \varepsilon \mathrm{yy}+\lambda \varepsilon \mathrm{zz} \\
& \sigma \mathrm{zz}=\lambda \varepsilon \mathrm{xx}+\lambda \varepsilon \mathrm{yy}+(\lambda+2 \mathrm{G}) \varepsilon \mathrm{zz} \\
& \sigma \mathrm{xy}=2 \mathrm{G} \varepsilon \mathrm{xy} \\
& \sigma \mathrm{yz}=2 \mathrm{G} \varepsilon \mathrm{yz} \\
& \sigma \mathrm{zx}=2 \mathrm{G} \varepsilon \mathrm{zx},
\end{aligned}
$$

em que , a constante de Lamé $(\lambda)$ pode ser expressa como:

$$
\lambda=v \mathrm{E} /(1+v)(1-2 v)
$$

e o módulo de elasticidade transversal por:

$$
\mathrm{G}=\mathrm{E} / 2(1+v)
$$

Em que:

"E" é o módulo de elasticidade longitudinal (YOUNG) do material,

" $v$ " é o coeficiente de Poisson.

Para materiais cujas propriedades variam com as direções, como os materiais reforçados por fibras ou laminados a frio, deve-se usar relações constitutivas apropriadas, com propriedades elásticas dependentes da direção.

O conjunto de equações 3 ... 5, representam as equações fundamentais para a elasticidade tridimensional.

Então, o deslocamento do extremo livre é dado por (considerando elemento barra) [15]:

$$
\mathrm{u}_{\mathrm{x}}=\mathrm{PL} / \mathrm{A} \mathrm{E}
$$

em que:

$\mathrm{u}_{\mathrm{x}}=$ Deslocamento do extremo livre;

$\mathrm{P}=$ Força aplicada;

$\mathrm{L}=$ Comprimento do material;

$\mathrm{A}=$ Área

\subsection{PASSOS PARA A ANÁLISE FEA}

A análise FEA pressupõe algumas etapas para a correta aplicação das condições de contorno, de acordo com Figura 4, quais sejam: Modelamento geométrico, Preparação das malhas, Definir simulação, Solver, Avaliar resultados e Otimização [10].

A etapa de modelamento geométrico corresponde a vetorização do desenho e posterior adição das álgebras booleanas, para a transformação em sólido de fato. Nesta etapa também está incluso o dimensionamento e os diversos acabamentos finais do modelo, como na realidade.

A etapa de preparação da malha, consiste em discretizar a estrutura, neste caso o modelo matemático anteriormente criado, em elementos paramétricos ou isoparamétricos conhecidos. 
Também é possível refinar a malha em alguma região de maior interesse, visando uma melhor acuracidade de resultados finais.

A etapa de definição da simulação, faz referência aos diversos tipos de simulações a serem aplicadas, a saber: Térmica, Modal, Estática, Dinâmica, CFD, entre outras.

A etapa do solver consiste nos cálculos internos executados pelo aplicativo CAE, neste caso o Abaqus, com base no algoritmo computacional de controle.

A etapa de avaliação dos resultados e otimização é o ultimo passo da analise FEA e consiste na avaliação dos resultados obtidos e sua adequada ponderação técnica, observando a função do produto e que foi objeto de analise.

Caso seja necessário, é possível fazer uma otimização estrutural ou topológica, visando a adequação das condições iniciais, impostas para os diversos tipos de carregamentos.

Para esta otimização, existe também a possibilidade de estabelecer um parâmetro para cada variável a ser analisada, de forma a obter uma resposta de sensibilidade variacional, de acordo com as boundary conditions, apresentadas como input na primeira etapa deste procedimento.

A Figura 4 ilustra melhor as diversas etapas da analise FEA.
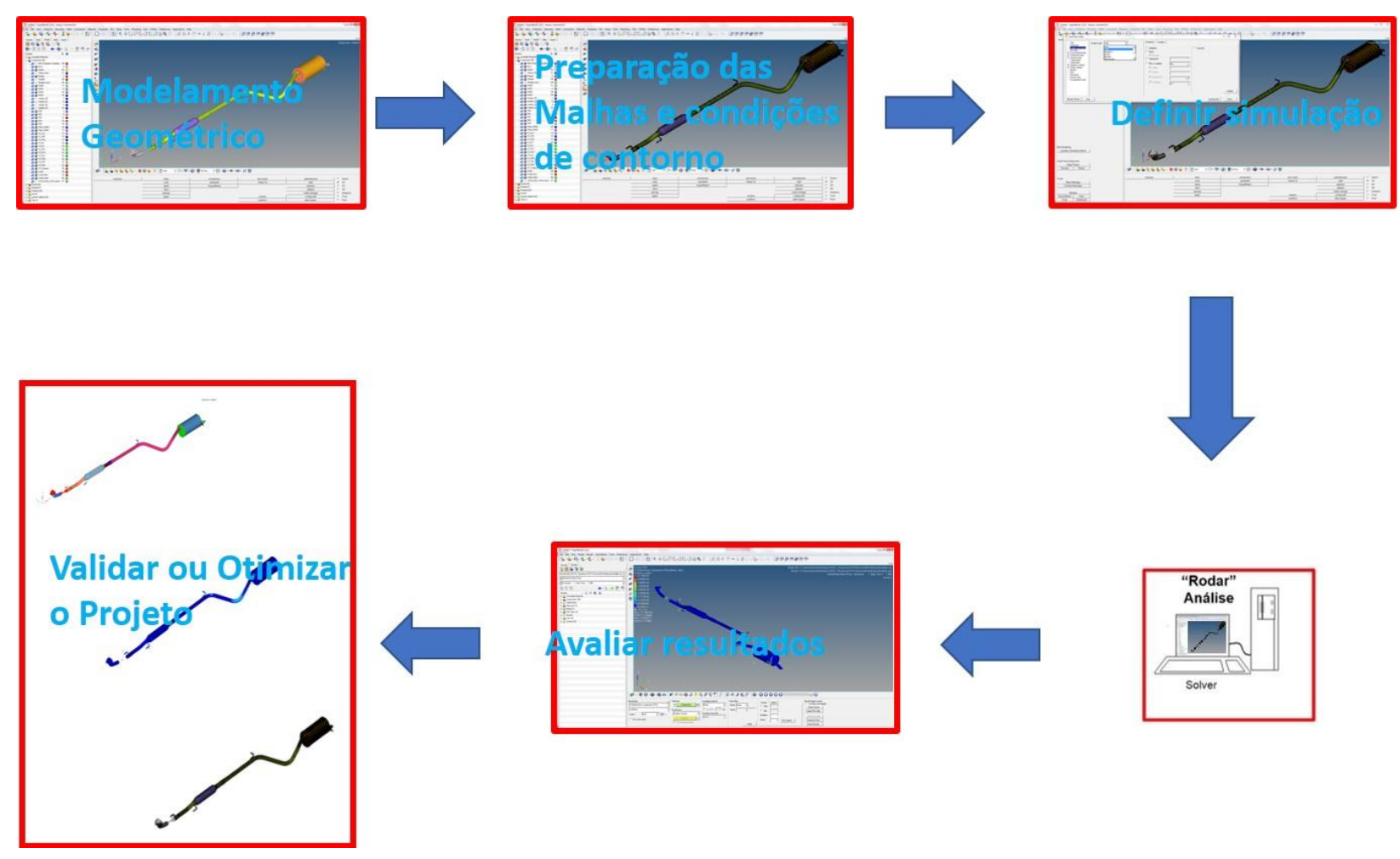

Figura 4 - Topologia computacional da analise FEA

\section{ESTUDO DE CASO: Análise das tensões considerando as frequências naturais}

A partir da simulação das condições de contorno, definidas como representativas da vida operacional do sistema de exaustão, e de posse do modelo matemático tridimensional em 
CAD, utilizou-se a técnica dos Elementos Finitos, como forma de prever o funcionamento deste componente junto ao conjunto.

Inicialmente foram geradas as malhas com o Hypermesh, considerando elementos do tipo "QUAD", de tamanho 5x5 para todo o sistema, permitindo que fossem gerados apenas 5\% de elementos triangulares.

Para as propriedades dos componentes, foram utilizados as propriedades de "SHELL", para os componentes que não possuem variação na espessura, propriedades de "SOLID" para os elementos 3D como solda e componentes forjados ou fundidos.

Para os componentes que têm a propriedade de amortecimento, como por exemplo os Rubbers e o flexível, foram utilizados as propriedades de massa, para simular o peso desses componentes, e elementos de mola com as informações de deformação elástica.

Para as forças atuantes, foram geradas acelerações de magnitude 5G nos eixos "X" e "Y" e de $10 \mathrm{G}$ no eixo "Z", tendo por base o plano cartesiano.

A Figura 5 ilustra as condições de contorno, para o produto que esta sendo analisado, evidenciando as gravity loads de $5 \mathrm{G}$ e $10 \mathrm{G}$.

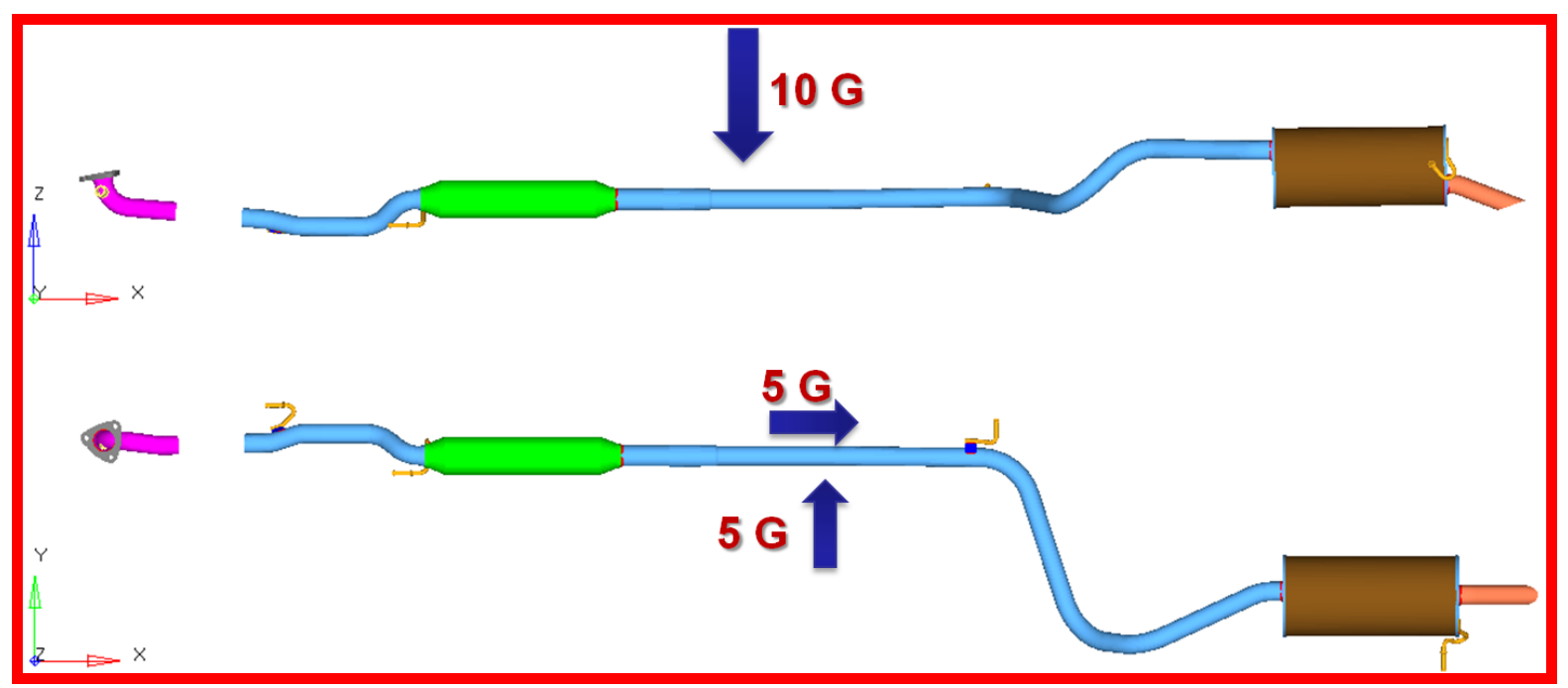

Figura 5 - Condições de contorno

Definidas as condições de contorno para o modelo matemático, o próximo passo foi o de configurar o solver, para a condição de análise estática, indicando as configurações de forças e engastes.

Com o solver devidamente configurado, deu-se inicio a análise computacional, em que o solver realizou os cálculos da análise estrutural, via interface do aplicativo ABAQUS. Após a finalização dos cálculos, o solver apresentou os resultados graficamente, indicando no modelo matemático, os deslocamentos e as tensões geradas no escapamento. 
A Figura 6 ilustra as tensões geradas no sistema de exaustão, visualizadas nas vistas de planta e elevação do conjunto.

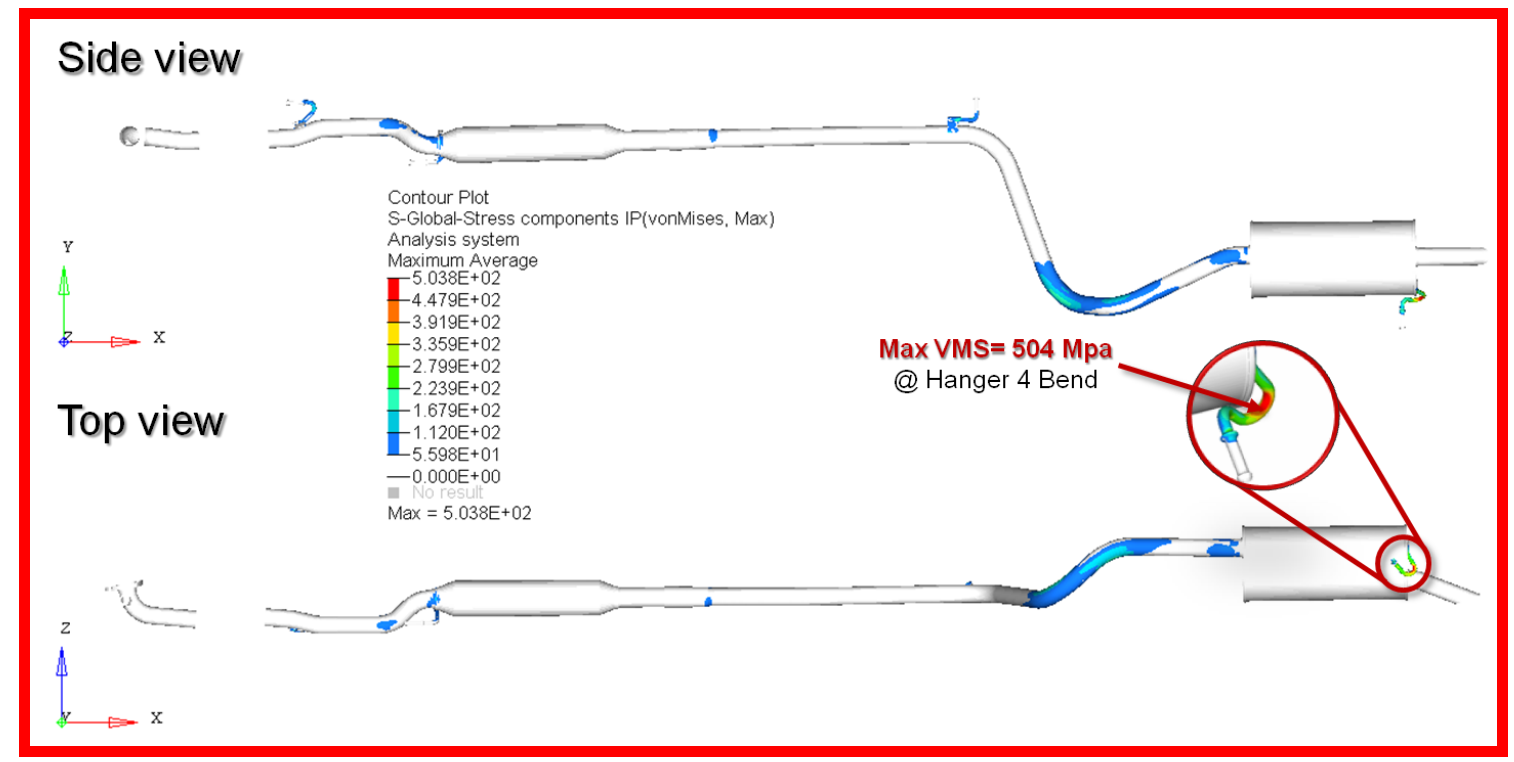

Figura 6 - Tensões de Von Mises (MPa)

De posse do resultado da análise, verificou-se a existência de uma região com uma concentração de tensão superior a 500MPa, ou seja, dentro da faixa considerada como um alerta de problema em potencial. Com estas informações, o engenheiro do produto poderá decidir por ações de modificações, na região com problema, evitando o desperdício de material e reforçando as áreas críticas, para o bom desempenho do conjunto de exaustão.

Desta forma, o problema inicial apontado é resolvido pela simulação computacional.

A Figura 7 ilustra o produto hanger e sua frequência natural crítica, denotando a região de maior concentração de tensão.

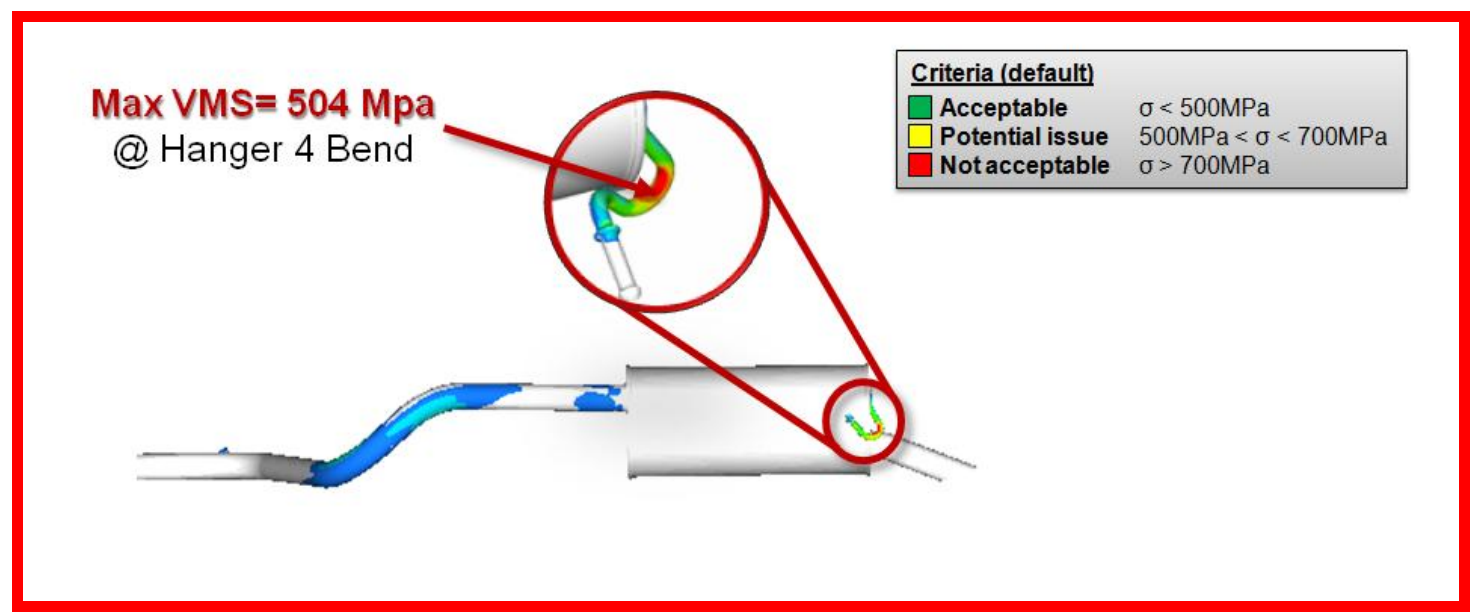

Figura 7 - Modos de Vibrações em cada hanger 
Com a finalização das análises, foi possível verificar se os componentes atendem as especificações do cliente, ou quais correções serão necessárias ainda na fase de prédesenvolvimento. Nesta fase, as cotações para definir o custo final do produto, ainda não foram congeladas, e desta forma, poderão ser realizadas com uma precisão maior, sem a duvida de que será necessário acrescentar material, ou adicionar mais algum componente, onerando o custo final do produto. Por outro lado, o protótipo será criado sem a necessidade de retrabalhos, reduzindo ainda mais o custo do produto.

\subsection{BULK DATA}

Para esta analise, utilizou-se um processador Intel Xeon W3565, 3.2 GHz, 64 bits, $10 \mathrm{~GB}$ RAM e disco rígido de 3,5 TB e 4 GB de swap_space, além de:

$\begin{array}{ll}\text { - Tempo de processamento total } & =09 \mathrm{~min} \\ \text { - No. de Elementos } & =137713 \\ \text { - No. de nós } & =143204 \\ \text { - No. de Variáveis } & =856176\end{array}$

\section{CONCLUSÃO}

O resultado da simulação indicou em qual região, e qual componente, seria necessário uma melhoria, para adequar o produto nas especificações do cliente. Todavia, para este estudo, o engenheiro do produto poderá verificar se estas tensões, realmente irão trazer danos ao produto, ou se irá diminuir a vida útil do mesmo. O motivo é que as concentrações de tensões, estão em uma faixa muito próxima ao limite inferior especificado. Esta faixa não indica uma reprovação do produto, mas sim, um ponto a ser verificado e posteriormente, analisado com maior profundidade, tendo como prerrogativa a função do produto.

Este resultado demonstra uma precisão e transparência para a cotação do valor final do produto, uma vez que é baseado em resultados do modelo CAD e simulação computacional. Assim, é previsto uma diminuição no tempo de reprojetos, e validações com construções de protótipos. Com este método, é possível mostrar ao cliente final, - neste caso a montadora que o produto oferecido parte de um projeto robusto, diminuindo a incerteza de problemas futuros, durante as fase de projeto.

\section{REFERÊNCIAS}

[1] Pahl, G., Beitz, W., Feldhusen, J., Grote, K.-H.: Projeto na Engenharia, $1^{\text {a }}$ ed., Edgard Blücher, São Paulo, 2005

[2] Ehrlenspiel, K., Kiewert, A., Lindemann, U.: Cost-Efficient Design, ASME Press, ISBN 0-7918-0250-7, New York, 2007

[3] Back, N., Ogliari, A., Dias, A., Da Silva, J.C.: Projeto Integrado de Produtos: Planejamento, Concepção e Modelagem, ISBN: 9788520422083, Editora Manole, 2008 
[4] Alves Filho, A.: Elementos Finitos - A Base da Tecnologia CAE, Editora Erica, $1^{\circ}$ Edição, São Paulo, 2000

[5] Timoshenko,S.P.: Mecânica dos Sólidos Vol. 1/2, Editora LTC, RJ, 1998

[6] Camargo, R.: CAE/CAD/CAM "Uma Ferramenta para Otimização de Projetos de Cames, Recursos e Aplicações de Cálculos pelo Método dos Elementos Finitos", UNIMEP, 1990

[7] MacNeal, Richard,H., Schwendler, W.: MSC/NASTRAN for Window, MacNeal Schwendler Corporation, San Diego / Califórnia, 1997

[8] Zienkiewicz, O. C. e Taylor, R. L.: Finite Element Method- Basic Formulation and Linear Problems, Vol.1, McGraw-Hill Co., New York, 1989

[9] Hughes, T.J.R.: Finite Element Method - Linear Static and Dynamic Finite Element Analysis, Prentice-Hall, Englewood Cliffs, 1987

[10] Camargo, R.: Topologia de Projeto Robusto aplicando o método dos Elementos Finitos, Revista Ciência e Tecnologia, v. VII, p. 13-29, 2004

[11] Cook, R. D.: Finite Element Modeling for Stress Analysis, J. Wiley, New York, 1995.

[12] Bathe, K. J.: Finite Element Procedures, Prentice-Hall, Englewood Cliffs, 1995

[13] Crisfield, M. A.: Finite Elements and Solution Procedures for Structural Analysis, Vol. 1: Linear Analysis, Pineridge Press, Swansea, UK, 1986.

[14] Fonseca, J.S.O, Selk, C.A.C., Pereira, L.T.V., Marczak, R.J., Alves, D.B., Luersen, M.A.: Ferramentas de Simulação em Mecânica: Elementos Finitos, UFRGS, 2002

[15] Rao, S.: Vibrações Mecânicas, Quarta Edição, Pearson, 2009 